\title{
Finite difference time domain analysis of bottom effect on sound propagation in shallow water
}

\author{
Kohji Futa ${ }^{1}$ and Toshiaki Kikuchi ${ }^{2}$ \\ ${ }^{1}$ Simulation Systems Division, MITSUBISHI Precision Co., Ltd. (MPC), \\ 345 Kami-machiya, Kamakura, 247-8505 Japan \\ ${ }^{2}$ Department of Earth and Ocean Sciences, National Defense Academy (NDA), \\ 1-10-20 Hashirimizu, Yokosuka, 239-8686 Japan \\ e-mail: kikuchi@cc.nda.ac.jp
}

( Received 20 December 2000, Accepted for publication 10 January 2001)

Keywords: Shallow water, FDTD method, Bottom effect, Sediment, Transient field PACS number: 43.30.Ma, 43.30.Pc, 43.20.Mv

\section{Introduction}

There is shallow water area around Japan whose depth is a few hundreds meters such as the East China Sea. In such a shallow water, interaction of sea surface and bottom is so strong that the ocean behaves like a waveguide for acoustic waves and the acoustic field becomes much more complex than in a deep ocean. In general, the acoustic field in an ocean is expressed as an acoustic pressure distribution which is calculated by normal mode (NM) methods or parabolic equation (PE) methods for a monotonic sound (a sound of a single frequency), or it is expressed as a pulse train calculated from frequency spectrum of the acoustic pressure of limited band width by using the inverse Fourier transformation. In these methods, we could get solutions for steady state and there are problems in analyzing transient phenomena. We study availability of the finite difference time domain (FDTD) method to analyzing transient characteristics of sound in the ocean. Iijima et al. reported that the FDTD method could be used for analysis of underwater acoustic field, that is originally being used for analysis of electromagnetic field $[1,2]$. In this paper, we focus on a transient acoustic field by a pulse source in a shallow ocean.

\section{Theoretical background}

In an orthogonal coordinate, $x, y$ is range and depth, respectively. In this coordinate, the relationship between particle speed and acoustic pressure is represented as following:

$$
\begin{aligned}
& -\frac{1}{K} \frac{\partial p}{\partial t}=\frac{\partial v_{x}}{\partial x}+\frac{\partial v_{y}}{\partial y}, \\
& -\rho \frac{\partial v_{x}}{\partial t}=\frac{\partial p}{\partial x}+\eta_{x} v_{x}, \\
& -\rho \frac{\partial v_{y}}{\partial t}=\frac{\partial p}{\partial y}+\eta_{y} v_{y},
\end{aligned}
$$

where $p$ is instantaneous acoustic pressure, $v_{x}, v_{y}$ are $x$ and $y$ components of particle speed of medium, $\rho$ is density of the medium, $K$ is bulk modulus of the medium, $t$ is time and $\eta_{x}$ and $\eta_{y}$ are drag coefficients that act on medium particles. We get a set of difference equations from these partial differential equations:

$$
\begin{aligned}
p^{n}(i, j)= & p^{n-1}(i, j) \\
-\frac{K \Delta t}{\Delta x}\left(v_{x}{ }^{n-1 / 2}(i+1 / 2, j)-v_{x}{ }^{n-1 / 2}(i-1 / 2, j)\right) & \\
-\frac{K \Delta t}{\Delta y}\left(v_{y}{ }^{n-1 / 2}(i, j+1 / 2)-v_{y}{ }^{n-1 / 2}(i, j-1 / 2)\right), & \\
v_{x}{ }^{n+1 / 2}(i+1 / 2, j)= & C_{v x 1} v_{x}{ }^{n-1 / 2}(i+1 / 2, j) \\
& -C_{v x 2}\left(p^{n}(i+1, j)-p^{n}(i, j)\right), \\
v_{y}{ }^{n+1 / 2}(i, j+1 / 2)= & C_{v y 1} v_{y}{ }^{n-1 / 2}(i, j+1 / 2) \\
& -C_{v y 2}\left(p^{n}(i, j+1)-p^{n}(i, j)\right),
\end{aligned}
$$

where

$$
\begin{aligned}
C_{v x 1} & =\left(1-\frac{\eta_{x} \Delta t}{2 \rho}\right) /\left(1+\frac{\eta_{x} \Delta t}{2 \rho}\right), \\
C_{v x 2} & =\left(\frac{\Delta t}{\rho \Delta x}\right) /\left(1+\frac{\eta_{x} \Delta t}{2 \rho}\right), \\
C_{v y 1} & =\left(1-\frac{\eta_{y} \Delta t}{2 \rho}\right) /\left(1+\frac{\eta_{y} \Delta t}{2 \rho}\right), \\
C_{v y 2} & =\left(\frac{\Delta t}{\rho \Delta y}\right) /\left(1+\frac{\eta_{y} \Delta t}{2 \rho}\right) .
\end{aligned}
$$

where $n$ is a temporal index, $i, j$ are spacial indices for $x, y$ coordinates, respectively.

\section{Result and consideration}

We assume a simple ocean model in which water depth is $150 \mathrm{~m}$ with a $150 \mathrm{~m}$-thick sediment layer showed in Fig. 1. The ocean surface is assumed to be a perfect free wall and the basement is a rigid wall. There are absorbing layers $(1 \mathrm{~dB} / \lambda)$ at both end of the ocean and Mur's absorbing boundary [3] in first order beyond the absorbing layers. Using this model, propagation of a pulse in Fig. 2 is calculated in case of three types of bottom in Table 1 .

Figure 3 shows a pressure distribution at 0.7 seconds after the source starts to transmit the pulse. The figure is drawn by a black and white color with a proper threshold pressure. In case of the bottom B and C, acoustic energy propagates in the sediment layer. Especially in case of bottom $\mathrm{C}$, the reflection 


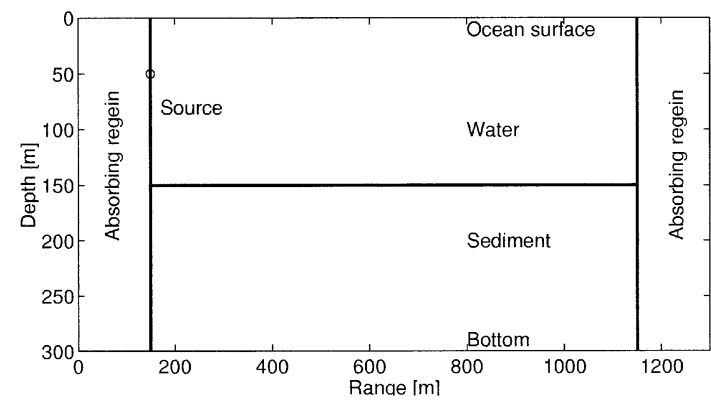

Fig. 1 Geometry of ocean model.

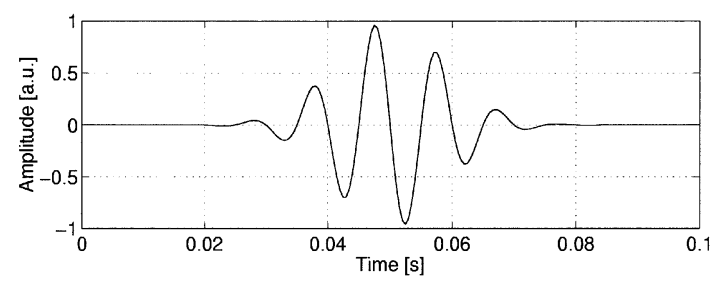

Fig. 2 Waveform of transmitted pulse.
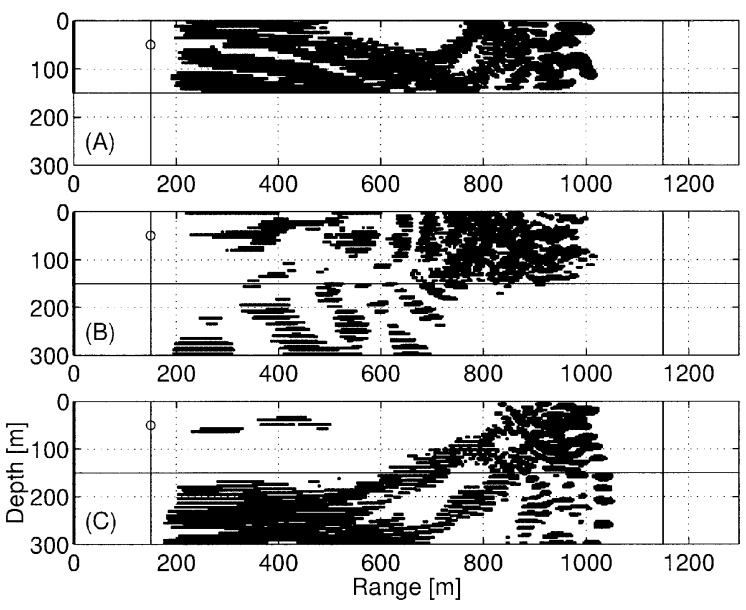

Fig. 3 Acoustic pressure distribution at $0.7 \mathrm{~s}$ after ping.

Table 1 Sound speed and density of the media.

\begin{tabular}{lccc}
\hline Medium & $c$ & $\rho$ & $\alpha$ \\
\hline Water & $1,500 \mathrm{~m} / \mathrm{s}$ & $1,000 \mathrm{~kg} / \mathrm{m}^{3}$ & $0 \mathrm{~dB} / \lambda$ \\
Bottom (A) & & - Rigid - & \\
Bottom (B) & $2,500 \mathrm{~m} / \mathrm{s}$ & $1,500 \mathrm{~kg} / \mathrm{m}^{3}$ & $0 \mathrm{~dB} / \lambda$ \\
Bottom (C) & $1,700 \mathrm{~m} / \mathrm{s}$ & $1,500 \mathrm{~kg} / \mathrm{m}^{3}$ & $0 \mathrm{~dB} / \lambda$ \\
\hline
\end{tabular}

by water-sediment interface is smaller and more acoustic energy goes into the sediment layer than in case of bottom B because an acoustic impedance contrast between water and sediment is low. (The contrast is 2.5 in B while 1.7 in C).

Figure 4 shows level of received signals with a $99 \mathrm{~m}$ deep receiver at the distance of $450 \mathrm{~m}$ from the sound source. When the sediment exists, pulses by low order modes are
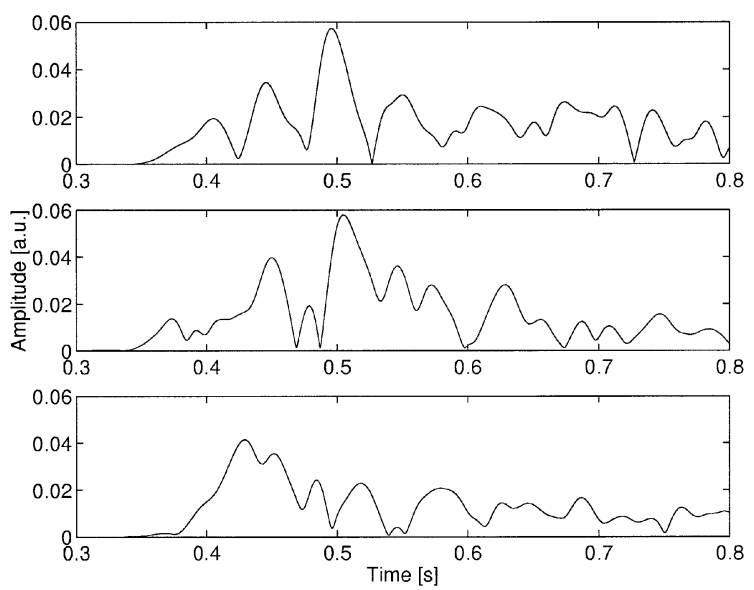

Fig. 4 Received signal of $99 \mathrm{~m}$ deep receiver.

(A)

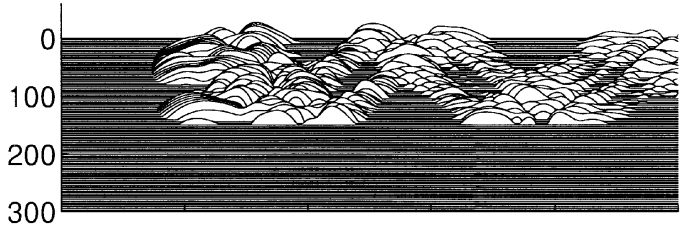

(B)
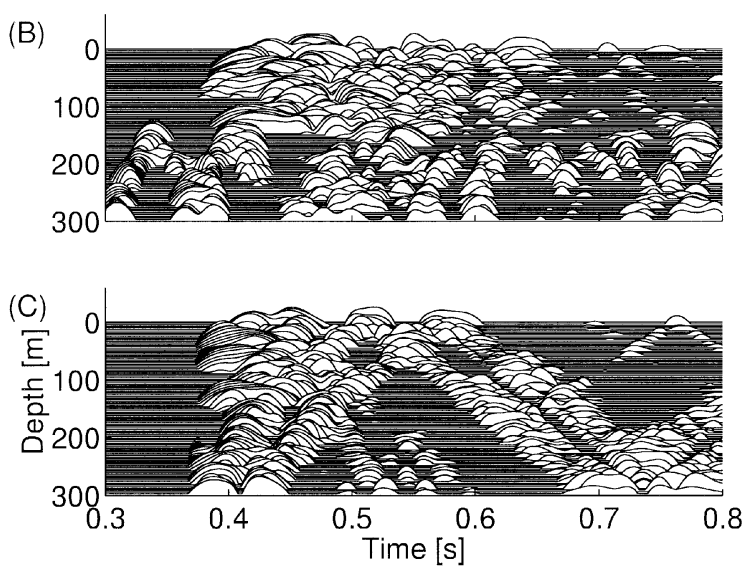

Fig. 5 Received signal at range of $450 \mathrm{~m}$.

dominant, while pulses by higher order modes can be observed for a long time in case of the rigid bottom.

Figure 5 shows time variation of vertical distribution of the acoustic pressure at the distance of $450 \mathrm{~m}$ from the sound source. In case of the rigid bottom, bottom A, there are a lot of pulses and the pattern of the plot seems to be a regular pattern. On the other hand, in case of sediment $\mathrm{C}$ that is low contrast in the acoustic impedance, we could think that the bottom depth become larger toward the basement and the effect of the basement become greater than in the case A. Therefore the pattern of vertical distribution of received pulses shows similar regularity but the number of pulses decreases. In case of relatively large impedance contrast bottom, B, the received pattern becomes complex because the acoustic energy is reflected by both the water-sediment interface and the basement, and the energy that travels fast in the sediment layer is observed. 


\section{Summary}

Sound propagation in shallow water is analyzed by using the FDTD method. By using the FDTD method, we can directly analyze the acoustic field with simple modeling, while the NM method needs complicated analysis in that not only discrete modes but also continuous modes have to be considered by using branch line integral, especially in the near field of a sound source. The FDTD method is efficient in analyzing the acoustic field near the sound source in time domain. Difference of the acoustic impedance of the sediment has a significant effect on the time-depth pattern of received acoustic pressure in pulse propagation in the shallow water. Consequently, it is found that the FDTD method is useful to analyze transient characteristics of acoustic field in a shallow ocean.

\section{References}

[1] F. Iijima, T. Tsuchiya, N. Endo and T. Tsuchiya, "Analysis of underwater acoustic propagation in ocean by FDTD method", Proc. Marine Acoust. Soc. Jpn. Meet., 107-110 (1999) (in Japanese).

[2] F. Iijima, T. Tsuchiya and N. Endo, "Analysis of underwater acoustic propagation in ocean by FDTD method (1)—Analysis of ASA benchmark problem-", Proc. Autumn Meet. Acoust. Soc. Jpn., 1155-1156 (1999) (in Japanese).

[3] O. Hashimoto and T. Abe, FDTD Jikan Ryouiki Sabunhou Nyuumon (Morikita Publishing, Tokyo, 1996), pp. 19-24 (in Japanese). 